\section{Peripheral Venous Catheter-Related Adverse Events in a Tropical Country}

Peripheral venous catheterization (PVC) is one of the most common procedures performed among hospitalized patients. However, $1 \%-10 \%$ of patients who undergo PVC develop local or systemic complications including phlebitis, infiltration, occlusion, tissue extravasation, and rarely, infection. ${ }^{1-6}$ The frequency of these complications may vary depending on the type of catheter, insertion site preparation, type of infusate, insertion technique, duration of catheterization, dressing type, and insertion site. ${ }^{1-6}$ Most studies evaluating PVC-related adverse events (PVCAEs) have been performed in United States and Europe. ${ }^{4,5,7-9}$ Generalization of these findings to tropical countries may be inappropriate given the high temperature and humidity, which may impact the duration of catheterization and the need for dressing changes. Data regarding PVCAEs in tropical countries are scarce. A prospective cohort study was conducted to evaluate the incidence, type, and associated factors of adverse events associated with PVCs.

From January 1, 2017, to March 31, 2017, 5 research nurses prospectively followed all hospitalized patients at Thammasat University Hospital, a 650-bed, tertiary hospital in Thailand. They also inspected and monitored all inserted PVCs daily for complications. From all hospitalized patients $>15$ years old with a PVC, consent for study participation was obtained. Each patient with a PVC in place for $>24$ hours was prospectively followed until PVC removal or complications occurred. Data collected included patient demographics, hospital location of PVC insertion(s), skin condition, Charlson comorbidity score, anatomical site of PVC, inserter, total duration of PVC and PVCAEs. Complications related to PVCs were categorized as mechanical or clinical PVCAEs. Each PVCAE was first recorded as a suspected event based on daily collection of the presence or absence of signs and/or symptoms during catheterization and up to 48 hours after catheter removal. A mechanical PVCAE was defined as any catheter dislodgement that was unplanned, an occlusion of the PVC, or a rupture of the closed infusion system when infusion lines were disconnected from the infusion bag or the absence of a well-sealed injection site. Clinical PVCAEs included localized edema at the site of insertion, phlebitis, hematoma at the insertion site, leakage of fluid/blood and suspected sepsis. Signs and symptoms recorded included redness and/or warmth, tenderness and/or pain, edema and/or swelling, induration, palpable venous cord, and presence of pus, hematoma, and/or fever (when concomitant with another symptom or sign). The grading for phlebitis was modeled from Maddox et al. ${ }^{10}$
Analyses were performed using SPSS software version 15 (IBM, Armonk, NY), and categorical data were compared using the 2 -tailed $\chi^{2}$ or Fisher's exact test, as appropriate. The MannWhitney $U$ test was used to compare continuous variables. Logistic regression was performed to assess predictors for outcomes (any PVCAEs). Adjustment was made for key variables including severity of illness, patient characteristics, settings of insertion, and anatomical of catheter insertions. Adjusted odd ratio (aOR) and 95\% confidence intervals (CIs) were computed; a significant statistical difference was defined as $P<.05$.

In total, 646 PVCs were placed in 500 hospitalized patients during the study period. The median age was 64 years (range, 49-76 years). The frequency and percentage of Charlson scores were as follows: 0-1 (254 of 500, 51.8\%), 2-3 ( 180 of $500,36.0 \%)$, and $>3$ (66 of $500,13.2 \%$ ). Overall, the median number of PVCs per patient was 1 (range, 1-7). Most PVCs were placed in medical units (58 of $646 ; 39.9 \%$ ) or surgical units (155 of $646,24 \%$ ), and PVCs were most commonly inserted into the hand $(271$ of $646,42 \%)$ or forearm (187 of $646,29 \%)$. The median dwelling time was 3 days (range, 1-24 days); 20.8\% of PVCAE patients (104 of 500) had a PVC $>3$ days. The overall PVCAE rate was 61.1 PVCAEs per 1,000 PVC days. The most common complications were occlusion (24.4 of 1,000 PVC days), phlebitis (19.1 of 1,000 PVC days), and PVC leakage (16 of 1, 000 PVC days) (Table 1). Mechanical PVCAEs were more common than clinical PVCAEs $(P=.01)$. Most PVCAEs occurred during the first 3 days after PVC insertion. There was no significant difference between time to mechanical PVCAE versus clinical PVCAE nor the rates of complications from different insertion sites. By multivariate analysis, being female (aOR, 1.6; 95\% CI, 1.083.36; $P=.02$ ), unstable PVC (aOR, 1.9; 95\% CI, 1.24-6.45; $P=.04$ ), and presence of cutaneous lesions (aOR, 2.2; 95\% CI, 1.45-5.67; $P=.01)$ were independent risk factors for any PVCAEs, while a covered transparent dressing (aOR, 0.78; 95\% CI, 0.55-0.92; $P=.02$ ) was protective.

Our findings highlight several key issues. First, despite the lower incidence of infection-related PVCAEs, the burden of PVCAEs in this tropical country was high compared to previously reported studies in nontropical countries. ${ }^{2,5-9}$ Second, the proportion of patients who had a PVC for $>3$ days was low, likely due to the high temperature and humidity. Although a recent recommendation suggested no benefit of routine PVC replacement (ie, between 72 and 96 hours), ${ }^{1}$ our data suggest that high temperature and humidity may have impacted on shorter duration of PVC replacement (Table 1). ${ }^{7}$ Third, our data emphasize the need for meticulous care of PVC during insertion and maintenance. To minimize the risk of occlusion, the PVC needs to be stabilized; a transparent dressing should be used for PVC maintenance.

This study has several limitations. First, given the nature of this pilot study, we did not collect clinical conditions (eg, sepsis), 
table 1. Patient Characteristics and Peripheral Venous CatheterAssociated Adverse Events (PVCAEs)

\begin{tabular}{|c|c|}
\hline Variable & $\begin{array}{l}\text { No. }(\%) \\
(\mathrm{N}=500)\end{array}$ \\
\hline Age, median y (range) & $64(49-76)$ \\
\hline Female & $256(51.2)$ \\
\hline \multicolumn{2}{|l|}{ Charlson's comorbidity score } \\
\hline $0-1$ & $254(51.8)$ \\
\hline $2-3$ & $180(36)$ \\
\hline$>3$ & $66(13.2)$ \\
\hline \multicolumn{2}{|l|}{ Skin condition } \\
\hline Healthy & $414(82.8)$ \\
\hline Lesions & $82(17.2)$ \\
\hline No. of PVCs, median (range) & $1(1-7)$ \\
\hline \multicolumn{2}{|l|}{ Anatomical site of insertion } \\
\hline Hand & $281(42)$ \\
\hline Forearm & $187(29)$ \\
\hline Anticubital & $86(17.2)$ \\
\hline Arm & $26(5)$ \\
\hline Leg & $52(10)$ \\
\hline Foot & $16(3)$ \\
\hline \multicolumn{2}{|l|}{ Hospital unit at insertion } \\
\hline Medicine & $200(40)$ \\
\hline Surgery & $124(24.8)$ \\
\hline Intensive care & $95(19)$ \\
\hline Others & $81(16.2)$ \\
\hline Documentation of dressing & $339(67.8)$ \\
\hline \multicolumn{2}{|l|}{ Type of dressing } \\
\hline Nonocclusive dressing & $36(7.2)$ \\
\hline Unclean dressing & $59(11.8)$ \\
\hline Covered transparent dressing & $405(81)$ \\
\hline Duration of PVC, median d (range) & $3(1-24)$ \\
\hline Total PVC days & 2,594 \\
\hline \multicolumn{2}{|c|}{ Incidence of PVCAEs per 1,000 PVC days } \\
\hline Mechanical & 45.96 \\
\hline Clinical & 27.52 \\
\hline \multicolumn{2}{|l|}{ PVCAE type per 1,000 PVC days } \\
\hline PVC-associated infection & 1.53 \\
\hline Phlebitis & 19.11 \\
\hline Hematoma & 6.88 \\
\hline Occlusion & 24.46 \\
\hline Dislodgement & 5.36 \\
\hline Leaking & 16.05 \\
\hline
\end{tabular}

NOTE. PVC, peripheral venous catheter.

nor did we assess all known risk factors associated with PVCAEs including type of infusate, catheter material, and type of cannula dressing. ${ }^{2,4-10}$ Second, our PVCAE definition was derived mainly from clinical parameters, and $>50 \%$ of the study population were elderly. Third, the small sample size in our study may have limited our capacity to identify other potential risk factors associated with PVCAEs. Despite these limitations, our study results suggest that the PVCAE burden is underestimated in this tropical country. Proper training for PVC insertion, maintenance, and monitoring after PVC insertion are crucial components of improving care to prevent PVCAEs in tropical countries with high humidity and temperature.

\section{ACKNOWLEDGMENT}

Financial support: No financial support was provided relevant to this article.

Potential conflicts of interest: All authors report no conflicts of interest relevant to this article.

\section{Anucha Apisarnthanarak, MD; ${ }^{1}$ Nalini Singh, MD, MPH; David J. Weber, MD, $\mathrm{MPH}^{3}$}

Affiliations: 1. Division of Infectious Diseases, Thammasat University Hospital, Pathumthani, Thailand; 2. Division of Infectious Diseases, Department of Pediatrics, Epidemiology, and Global Health, School of Medicine and Health Sciences, School of Public Health, Children's National Medical Center, George Washington University, Washington DC; 3. Gillings School of Public Health, University of North Carolina, Chapel Hill, North Carolina.

Address correspondence to Anucha Apisarnthanarak, MD, Division of Infectious Diseases, Thammasat University Hospital, Pratumthani, Thailand, 12120 (anapisarn@yahoo.com).

Received April 29, 2017; accepted July 5, 2017; electronically published August 10, 2017

Infect Control Hosp Epidemiol 2017;38:1258-1259

(c) 2017 by The Society for Healthcare Epidemiology of America. All rights reserved. 0899-823X/2017/3810-0020. DOI: 10.1017/ice.2017.164

\section{REFERENCES}

1. Gorski LA, Dora H, Kuehn SC. Recommendation for frequency of assessment of the short peripheral catheter site. J Infus Nurs 2012;35:290-292.

2. Abolfotouh MA, Salam M, Bani-Mustafa A, White D, Balkhy $\mathrm{HH}$. Prospective study of incidence and predictors of peripheral intravenous catheter-induced complications. Ther Clin Risk Manag 2014;10:993-1001.

3. Soifer NE, Borzak S, Edlin BR, Weinstein RA, Reese M. Prevention of peripheral venous catheter complications with an intravenous therapy team. Arch Intern Med 1998;158:473-477.

4. Maki DG, Kluger DM, Crnich CJ. The risk of bloodstream infection in adults with different intravascular devices: a systematic review of 200 published prospective studies. Mayo Clin Proc 2006;81:1159-1171.

5. Trinh TT, Chan PA, Edwards O, et al. Peripheral venous catheterrelated Staphylococcus aureus bacteremia. Infect Control Hosp Epidemiol 2011;32:579-583.

6. Lee W-LL, Chen H-LL, Tsai T-YY, et al. Risk factors for peripheral intravenous catheter infection in hospitalized patients: a prospective study of 3165 patients. Am J Infect Control 2009;37:683-686.

7. Rickard CM, McCann D, Munnings J, McGrail MR. Routine resite of peripheral intravenous devices every 3 days did not reduce complications compared with clinically indicated resite: a randomized controlled trial. BMC Medicine 2010;8:53.

8. Rickard CM, Webster J, Wallis MC, et al. Routine versus clinically indicated replacement of peripheral intravenous catheters: a randomized controlled equivalence trial. Lancet 2012;380: 1066-1074.

9. Webster J, Clarke S, Paterson D, et al. Routine care of peripheral intravenous catheters versus clinically indicated replacement: randomized controlled trial. BMJ 2008;337:a339.

10. Maddox RR, Rush DR, Rapp RP, Foster TS, Mazella V, McKean HE. Double-blind study to investigate methods to prevent cephalothin-induced phlebitis. Am J Hosp Pharm 1977;34:29-34. 